\title{
COERCIVENESS OF THE NORMAL BOUNDARY PROBLEMS FOR AN ELLIPTIC OPERATOR
}

\author{
BY GERD GRUBB
}

\author{
Communicated by Avner Friedman, July 24, 1969
}

Let $\Omega$ be a bounded open subset of $R^{n}$, with smooth boundary $\Gamma$ (the theory is easily extended to compact manifolds). Let $A$ be a differential operator of order $2 m(m \geqq 1)$, with coefficients in $C^{\infty}(\bar{\Omega})$, such that $A$ is uniformly strongly elliptic and formally selfadjoint in $\bar{\Omega}$. We consider the $L^{2}(\Omega)$-realizations of $A$, determined by boundary conditions of the form

$$
\gamma_{j} u-\sum_{k \in K, k<j} F_{j k} \gamma_{k} u=0, \quad j \in J
$$

here $J$ and $K$ are complementing subsets, each consisting of $m$ elements, of the set $M=\{0, \cdots, 2 m-1\}$; the $F_{j k}$ denote (pseudo-) differential operators in $\Gamma$ of orders $j-k$; and the $\gamma_{k}$ denote the standard boundary operators: $\gamma_{0} u=\left.u\right|_{\Gamma}, \gamma_{k} u=\left.D_{n}^{k} u\right|_{\Gamma}$, for $u \in C^{\infty}(\bar{\Omega})$, where $i D_{n}=\partial / \partial n$ is the interior normal derivative at $\Gamma$. (1) is a reduced form of the usual normal type of boundary conditions, generalized to include pseudo-differential operators in $\Gamma$.

Let $\tilde{A}$ be the operator in $L^{2}(\Omega)$ defined by

$$
\begin{aligned}
D(\tilde{A}) & =\left\{u \in L^{2}(\Omega) \mid A u \in L^{2}(\Omega), u \text { satisfies }(1)\right\}, \\
\tilde{A} u & =A u \text { on } D(\tilde{A}) .
\end{aligned}
$$

(The definition is given a sense by the general concept of boundary value introduced by Lions-Magenes [7]). We shall give below a necessary and sufficient condition on the operators $F_{j k}$ (together with $A$ ) in order that $\tilde{A}$ be $m$-coercive, i.e. satisfies

$$
\operatorname{Re}(\tilde{A} u, u)+\lambda\|u\|_{0}^{2} \geqq c\|u\|_{m}^{2}, \quad \forall u \in D(\tilde{A}),^{1}
$$

for some $c>0, \lambda \in R$. The condition has two parts:

$1^{\circ}$ it is necessary that the $F_{j k}$ with $j$ and $k \geqq m$ are certain functions of the $F_{j k}$ with $j$ and $k<m$ in order that $\tilde{A}$ be even lower bounded (Theorem 1);

AMS Subject Classifications. Primary 3545, 3519, 3523; Secondary 3504.

Key Words and Phrases. Coerciveness inequality, normal boundary conditions, elliptic operator, formally selfadjoint, nonselfadjoint, lower bounded realization, variational, pseudo-differential operator, principal symbol.

${ }^{1}$ Here $\|u\|$. denotes the norm in the Sobolev space $H^{\circ}(\Omega), s \in R$. 
$2^{\circ}$ when $1^{\circ}$ is fulfilled, the $m$-coerciveness is equivalent with an algebraic condition on the principal symbols (Theorem 2). Theorems 1-2 arise as corollaries of a general result (Theorem 3), which permits application of [4], [5].

In [1], Agmon gave an algebraic condition for $m$-coerciveness of selfadjoint realizations defined by differential boundary operators; restricted to such realizations, our condition is equivalent with his. Our result also extends those of Fujiwara-Shimakura [3] and Grubb [5], treating certain nonselfadjoint classes of (1). The theory avoids the classical considerations of integro-differential forms, which are not very convenient for the question of necessity. However, our $m$-coercive $\tilde{A}$ are variational in the sense of [5] (i.e., $\tilde{A}+\lambda$ is regularly accretive in Kato's sense, for suitable $\lambda \in R$ ).

1. A necessary condition for lower boundedness. For a $(p \times q)$

$$
E=\left(\left(E_{j k}\right)\right)_{\substack{j=0, \ldots, p-1 \\ k=0, \cdots, q-1}}
$$

and two ordered subsets $N_{1}$ and $N_{2}$ of $\{0, \cdots, p-1\}$ resp. $\{0, \cdots, q-1\}$, we denote the minor $\left(\left(E_{j k}\right)\right)_{j \in N_{1}, k \in N_{2}}$ by $E_{N_{1} N_{2}}$. Similarly for a row- or column-vector $\phi=\left\{\phi_{0}, \cdots, \phi_{p-1}\right\}$ we denote $\left\{\phi_{j}\right\}_{j \in N_{1}}$ by $\phi_{N_{1}}$. We also use $\phi_{N_{1}}$ to indicate a vector $\left\{\phi_{j}\right\}_{j \in N_{1}}$ indexed by $N_{1}$.

Let $J, K$ and $M$ be as above, then we introduce the ordered subsets of $M: M_{0}=\{0, \cdots, m-1\}, M_{1}=\{m, \cdots, 2 m-1\}, J_{0}=J \cap M_{0}$, $J_{1}=J \cap M_{1}, K_{0}=K \cap M_{0}$ and $K_{1}=K \cap M_{1}$. When $N \subset M$ we set $N^{\prime}=\{n \mid 2 m-1-n \in N\}$, considered again as an ordered subset of $M$. by $\rho$.

The "Cauchy" boundary operator $\left\{\gamma_{0}, \cdots, \gamma_{2 m-1}\right\}$ will be denoted

With this notation, (1) is equivalent with

$$
\rho_{J_{0}} u=F_{0} \rho_{K_{0}} u, \quad \rho_{J_{1}} u=F_{1} \rho_{K_{0}} u+F_{2} \rho_{K_{1}} u,
$$

where $F_{0}, F_{1}$ and $F_{2}$ are the matrices of (pseudo-)differential operators (where we put $F_{j k}=0$ for $\left.j \leqq k\right): F_{0}=\left(\left(F_{j k}\right)\right)_{j \in J_{0}, k \in K_{0}}, F_{1}=\left(\left(F_{j k}\right)\right)_{j \in J_{1}, k \in K_{0}}$ and $F_{2}=\left(\left(F_{j k}\right)\right)_{j \in J_{1}, k \in K_{1}}$. (Evident modifications when empty index sets occur.) They are of types $(-k,-j)_{j \in J_{0}, k \in K_{0}},(-k,-j)_{j \in J_{1}, k \in K_{0}}$ and $(-k,-j)_{j \in J_{1}, k \in K_{1}}$, respectively. (The notion of type is a convenient generalization of order to matrices, the principal symbol $\sigma^{0}$ is defined accordingly, see Hörmander [6], or [5].) Note the way in which $F_{0}$ and $F_{2}$ are minors of matrices with zeroes in and above the diagonal; we shall say that they are subtriangular.

The operator $F_{0}$, which maps $\prod_{k \in K_{0}} H^{s-k}(\Gamma)$ into $\prod_{k \in J_{0}} H^{s-k}(\Gamma)$, 
all $s \in R$, can be supplemented with the identity on $\prod_{k \in K_{0}} H^{s-k}(\Gamma)$ to yield an operator $\Phi$ from $\prod_{k \in K_{0}} H^{s-k}(\Gamma)$ to $\prod_{k \in M_{0}} H^{s-k}(\Gamma)$ :

$$
\Phi: \phi_{K_{0}} \mapsto \psi_{M_{0}}, \quad \text { where } \psi_{K_{0}}=\phi_{K_{0}}, \quad \psi_{J_{0}}=F_{0} \phi_{K_{0}} .
$$

We write in short

$$
\Phi=\left(\begin{array}{c}
I_{K_{0}} \\
F_{0}
\end{array}\right), \quad \text { where } I_{K_{0}}=\left(\left(\delta_{j k}\right)\right)_{j, k \in K_{0}} .
$$

The adjoint $\Phi^{*}$ sends $\phi_{M_{0}}$ into $\phi_{K_{0}}+F_{0}^{*} \phi_{J_{0}}$ and is written in short as $\Phi^{*}=\left(I_{K_{0}} F_{0}^{*}\right) . \Phi$ and $\Phi^{*}$ are (pseudo-)differential operators of types $(-k,-j)_{j \in M_{0}, k \in K_{0}}$ resp. $(k, j)_{j \in K_{0}, k \in M_{0}}$; with an analogous notation for their symbols one has e.g. $\sigma^{0}\left(\Phi^{*}\right)=\left(I_{K_{0}} \sigma^{0}\left(F_{0}\right)^{*}\right)$.

At the points of $\Gamma$ one may write $A$ in normal and tangential coordinates

$$
A=\sum_{l=0}^{2 m} A_{l} D_{n}^{l}
$$

where the $A_{l}$ denote differential operators in $\Gamma$ of orders $2 m-l ; A_{2 m}$ is a positive function. Then one has the Green's formula

$$
(A u, v)-(u, A v)=\int_{\Gamma} a \rho u \cdot \overline{\rho v} d \sigma, \quad u, v \in C^{\infty}(\bar{\Omega}),
$$

where $Q$ is a $(2 m \times 2 m)$-matrix of differential operators in $\Gamma$ : $Q$ $=\left(\left(Q_{j k}\right)\right)_{j, k \in M}$ where each $\mathfrak{Q}_{j k}$ has the form $i A_{j+k+1}+$ differential operators of orders less than $2 m-\left(j+k+1\right.$ ) (we put $A_{l}=0$ for $l>2 m$ ), cf. Seeley [8], or [5]. We note that $Q^{*}=-a$, and that $Q$ is skewtriangular and invertible with $Q^{-1}$ a differential operator; $a$ is elliptic of type $(-k,-2 m+j+1)_{j, k \in M}$.

THEOREM 1. If $\tilde{A}$ is lower bounded, that is, if there exists $\lambda \in R$ such that $\operatorname{Re}(\tilde{A} u, u) \geqq \lambda\|u\|_{0}^{2}, \forall u \in D(\tilde{A})$, then $K_{0}=J_{1}{ }^{\prime}$, and

$$
F_{2}=-\left(\Phi^{*} Q_{M_{0} J_{1}}\right)^{-1} \Phi^{*} Q_{M_{0} K_{1}}
$$

(Here $\Phi^{*} \propto_{M_{0} J_{1}}$ is invertible when $K_{0}=J_{1}^{\prime}$, thanks to the special character of $Q$ and the subtriangularity of $F_{0}$.)

REMARK 1. The case treated by Fujiwara-Shimakura [3], Fujiwara [2] and Grubb $[5,4.3-4.4]$ is the case where

$$
K_{0}=J_{1}^{\prime}=\{m-p, \cdots, m-1\}
$$

for some $p \leqq m$, here $F_{0}$ and $F_{2}$ are 0 by their subtriangularity; the case in Grubb $[5,4.5]$ takes general $K_{0}$ but $F_{0}=0$. 
2. The condition for $m$-coerciveness. In accordance with (6), the principal symbol of $A$ may at points $y \in \Gamma$ be written in the form $a(y, \eta, \tau)=\sum_{l=0}^{2 m} a^{l}(y, \eta) \tau^{l}$, where $a_{l}(y, \eta)$ denotes the principal symbol of $A_{l}$; here $\eta$ belongs to the fibre at $y$ of the cotangent bundle $T^{*}(\Gamma)$, and $\tau \in R$. For each $(y, \eta)$ with $\eta \neq 0$, the polynomial $a(y, \eta, \tau)$ has exactly $m$ roots $\left\{\tau_{i}^{+}(y, \eta)\right\}_{i=1}^{m}$ in $\{\lambda \in C \mid \operatorname{Im} \lambda>0\}$. We can then form the polynomial $\prod_{i=1}^{m}\left(\tau-\tau_{i}^{+}(y, \eta)\right)=\sum_{l=0}^{m} s_{l}(y, \eta) \tau^{l}$, and use the coefficients to define the following $(m \times m)$-matrix valued functions on the nonzero subbundle $T_{\bullet}^{*}(\Gamma)$ of $T^{*}(\Gamma): S_{0}(y, \eta)=\left(\left(s_{k-j}(y, \eta)\right)\right)_{j, k \in M_{0}}$ and $S_{m}(y, \eta)=\left(\left(s_{m+k-j}(y, \eta)\right)\right)_{j, k \in M_{0}}$, where we put $s_{l}=0$ for $l \notin[0, m]$. Denoting by $I^{\times}$the skew-unit matrix $\left(\left(\delta_{j, m-1-k}\right)\right)_{j, k \in M_{0}}$, we finally introduce $Q=i I \times \bar{S}_{m} S_{m}, R=i I \times \bar{S}_{m} S_{0}$, here $\bar{S}$ denotes the complex conjugate of $S$. (More details in [5, Chapter 4], in fact $Q=A_{2 m}^{-1} \sigma^{0}\left(Q_{M_{0} M_{1}}\right)$, and $R$ is the principal symbol of a certain pseudo-differential operator in $\Gamma$.)

Theorem 2. $\tilde{A}$ is $m$-coercive if and only if it satisfies (i) and (ii):

(i) $K_{0}=J_{1}{ }^{\prime}$, and $F_{2}=-\left(\Phi * a_{M_{0} J_{1}}\right)^{-1} \Phi^{*} a_{M_{0} K_{1}}$.

(ii) Let $J_{2}=\left\{j \mid j+m \in J_{1}\right\}$, and let $E(y, \eta)$ be the matrix valued function on $T_{*}^{*}(\Gamma)$ :

$$
E=\sigma^{0}(\Phi) * Q_{M_{0} J_{2}} \sigma^{0}\left(F_{1}\right)+\sigma^{0}(\Phi) * R \sigma^{0}(\Phi),
$$

then $E+E^{*}$ is positive definite on $T^{*}(\Gamma)$.

In the affirmative case, $\tilde{A}$ is $2 m$-regular $\left(\tilde{A} u \in H^{s}(\Omega) \Rightarrow u \in H^{s+2 m}(\Omega)\right.$, $\forall s \geqq 0$ ), and $\tilde{A}^{*}$ is also $m$-coercive and $2 m$-regular.

3. Explanations and further developments. The first step in our proof of Theorems $1-2$ is the transformation of (4) into an equivalent boundary condition of the form

$$
\gamma_{J_{0}} u=F_{0} \gamma_{K_{0}} u, \quad \chi_{J_{1}} u=G_{1} \gamma_{\kappa_{0}} u+G_{2} \chi_{\kappa_{1}} u,
$$

where $\gamma$ and $\chi$ denote the $m$-vectors of boundary operators: $\gamma=\rho_{M_{0}}, \chi$

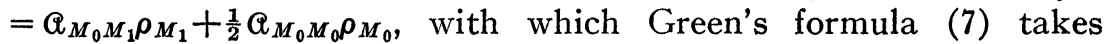
the simple form: $(A u, v)-(u, A v)=\int_{\Gamma}(\chi u \cdot \bar{\gamma} \bar{v}-\gamma u \cdot \bar{\chi} \bar{v}) d \sigma$. Note that $\chi=\left\{\chi_{k}\right\}_{k \in M_{0}}$, where $\chi_{k}$ is of order $2 m-k-1$. There is 1-1 correspondence between the systems $\left(F_{0}, F_{1}, F_{2}\right)$ and $\left(F_{0}, G_{1}, G_{2}\right)$ (we omit the formulae); $G_{2}^{*}$ is again subtriangular.

Assuming, as we may, that the Dirichlet problem for $A$ is uniquely solvable, we define the operator $P_{\gamma, x}$ in $D^{\prime}(\Gamma)^{m}$ by: $P_{\gamma, \chi} \phi=\chi z$, where $z$ is the solution of $A z=0$ in $\Omega, \gamma z=\phi$ (cf. [4], [5]). $P_{\gamma, x}$ is a selfadjoint pseudo-differential operator in $\Gamma$ of type $(-k,-2 m+j+1)_{j, k \in M_{0}}$ (Vainberg-Grušin [9]); its principal symbol is described in detail in [5, Chapter 4]. 
THEOREM 3. In addition to the notations introduced above, let $\Psi$ be the operator analogous to $\Phi$ with $F_{0}$ replaced by $-G_{2}^{*}$. Let $X$ $=\Phi\left(\prod_{k \in k_{0}} H^{-k-1 / 2}(\Gamma)\right)$ and let $Y=\Psi\left(\prod_{k \in J_{1}} H^{-k-1 / 2}(\Gamma)\right)$. Let $\Phi_{1}$ and $\Psi_{1}$ be the restrictions of $\Phi$ and $\Psi$ with domains $\prod_{k \in K_{0}} H^{-k-1 / 2}(\Gamma)$ resp. $\prod_{k \in J_{1}}, H^{-k-1 / 2}(\Gamma)$ and ranges $X$ resp. $Y$, clearly they are isomorphisms. Finally, introduce the pseudo-differential operator $\mathfrak{L}_{1}$ of type $(-k,-2 m+j+1)_{j \in J_{1}{ }^{\prime} k \in K_{0}}$ :

$$
\mathfrak{L}_{1}=G_{1}-\Psi^{*} P_{\gamma, \kappa} \Phi .
$$

Then $\tilde{A}$ corresponds, in the sense of $[4$, Theorem III 2.1] (based on the Dirichlet problem), to the operator $L: X \rightarrow Y^{\prime}$ defined by

$$
\begin{aligned}
D(L) & =\left\{\phi \in X \mid \mathfrak{L}_{1} \Phi_{1}^{-1} \phi \in \prod_{k \in J_{1}^{\prime}} H^{k+1 / 2}(\Gamma)\right\}, \\
L \phi & =\left(\Psi_{1}^{*}\right)^{-1} \mathscr{L}_{1} \Phi_{1}^{-1} \phi, \quad \text { when } \phi \in D(L) .
\end{aligned}
$$

Theorem 1 follows from this by use of [4, Theorem III 4.3]: Lower boundedness of $\tilde{A}$ implies $X \subset Y$, and then by the subtriangularity $\Phi=\Psi$, so that $K_{0}=J_{1}{ }^{\prime}$ and $F_{0}=-G_{2}{ }^{*}$, which leads to (8). Note that then $X=Y$.

Theorem 2 uses [5, Corollary 2.4]: $\tilde{A}$ is $m$-coercive if and only if $L$ is $m$-coercive, i.e., $X \subset Y$ and $\exists c>0, \lambda \in R$ so that

$$
\operatorname{Re}\left\langle L \phi_{Y}, \phi_{Y^{\prime}}\right\rangle+\lambda\|\phi\|^{2}{ }_{\{-k-1 / 2\}} \geqq c\|\phi\|^{2}\{m-k-1 / 2\} \text { on } D(L) .{ }^{2}
$$

This is equivalent with a similar property for $L_{1}=\Psi_{1}^{*} L \Phi_{1}$, which is a certain "realization" of $\mathcal{L}_{1}$, and here the property amounts (besides $\Phi=\Psi$ ) to the positive definiteness of $\sigma^{0}\left(\mathfrak{L}_{1}+\mathfrak{L}_{1}^{*}\right.$ ) (in fact $\left.E=A_{2 m}^{-1} \sigma^{0}\left(\mathscr{L}_{1}\right)\right)$; the computations resemble those in [5]. The last statement in Theorem 2 uses the ellipticity of $\mathfrak{L}_{\mathbf{1}}$.

REMARK 2 . The selfadjoint $m$-coercive $\tilde{A}$ are characterized by Theorem 2 (i), (ii), plus selfadjointness of $G_{1}=\Phi^{*} Q_{M_{0} J_{1}} F_{1}+\frac{1}{2} \Phi^{*} a_{M_{0} M_{0}} \Phi$ (then $E$ is also selfadjoint).

REMARK 3. Theorem 3 gives a basis for the discussion of many other properties of $\tilde{A}$, because of the way in which they are preserved by the correspondence between $\tilde{A}$ and $L$, see [4], [5]. Regarding coerciveness, we mention that:

$1^{\circ}$ the conditions in Theorem 2 are also necessary and sufficient for $(m-\epsilon)$-coerciveness with $\epsilon \in[0,1 / 2$ [ (cf. Fujiwara-Shimakura [3]),

$2^{\circ}$ the discussion of $(m-1 / 2)$-coerciveness in Fujiwara [2] (related to subellipticity [6]) seems extendable to the present case,

$2\|\phi\|_{\{0-k-1 / 2\}}$ denotes the norm in $\Pi_{k \in M_{0}} H^{\sigma-k-1 / 2}(\Gamma)$. 
$3^{\circ}$ a necessary condition for lower boundedness ("0-coerciveness") is the positive semidefiniteness of $\sigma^{0}\left(\mathfrak{L}_{1}+\mathfrak{L}_{1}^{*}\right)$ (cf. [5, Theorem 4.3]). Let us mention that lower boundedness $+2 m$-regularity do not imply $m$-coerciveness as in the selfadjoint case; examples using pseudodifferential operators: take $\mathfrak{L}_{1}$ elliptic with $\mathfrak{L}_{1}^{*}=-\mathfrak{L}_{1}$.

Concerning extensions of the results to operators $A$ that are merely strongly elliptic, let us mention that the case $K_{0}=J_{1}{ }^{\prime}$ $=\{m-p, \cdots, m-1\}$ has been treated by Fujiwara [2]; the device of [2] does not extend to our general case.

\section{REFERENCES}

1. S. Agmon, On the eigenfunctions and on the eigenvalues of general elliptic boundary value problems, Comm. Pure Appl. Math. 15 (1962), 119-147. MR 26 \#288.

2. D. Fujiwara, On some homogeneous boundary value problems bounded below, Proc. Japan Acad. 45 (1969).

3. D. Fujiwara and N. Shimakura, Sur les problèmes aux limites stablements variationnels, J. Math. Pures Appl. (to appear).

4. G. Grubb, $A$ characterization of the non-local boundary value problems associated with an elliptic operator, Ann. Sci. Norm. Sup. Pisa 22 (1968), 425-513.

5. - Les problèmes aux limites généraux d'un opérateur elliptique, provenants de la theorie variationnelle, Bull. Sci. Math. France (to appear).

6. L. Hörmander, Pseudo-differential operators and non-elliptic boundary problems, Ann. of Math. (2) 83 (1966), 129-209. MR 38 \#1387.

7. J. L. Lions and E. Magenes, Problèmes aux limites non homogènes et applications. Vol. 1, Dunod, Paris, 1968.

8. R. T. Seeley, Singular integrals and boundary value problems, Amer. J. Math. 88 (1966), 781-809. MR 35 \#810.

9. B. R. Vainberg and V. V. Grušin, Uniformly nonelliptic problems. II, Math. Sb. 73 (115) (1967), 126-154 = Math. USSR Sb. 2 (1967), 111-133. MR 36 \#552.

University of Copenhagen, DenMark 